\title{
(6) OPEN ACCESS \\ Soluble urokinase plasminogen activator receptor (suPAR) in acute care: a strong marker of disease presence and severity, readmission and mortality. A retrospective cohort study
}

\author{
Line Jee Hartmann Rasmussen, ${ }^{1}$ Steen Ladelund, ${ }^{1}$ Thomas Huneck Haupt, ${ }^{1}$ \\ Gertrude Ellekilde, ${ }^{2}$ Jørgen Hjelm Poulsen, ${ }^{3}$ Kasper Iversen, ${ }^{4}$ Jesper Eugen-Olsen, ${ }^{1}$ \\ Ove Andersen ${ }^{1}$
}

\section{- Additional material is published online only. To view please visit the journal online (http://dx.doi.org/10.1136/ emermed-2015-205444). \\ ${ }^{1}$ Clinical Research Centre, Copenhagen University Hospital Hvidovre, Hvidovre, Copenhagen, Denmark ${ }^{2}$ Acute Medical Department, Copenhagen University Hospital Hvidovre, Hvidovre Copenhagen, Denmark ${ }^{3}$ Department of Clinical Biochemistry, Copenhagen University Hospital Hvidovre, Hvidovre, Copenhagen, Denmark \\ ${ }^{4}$ Department of Cardiology, Copenhagen University Hospital Herlev, Herlev, Copenhagen, Denmark}

\section{Correspondence to} Line Jee Hartmann Rasmussen, Clinical Research Centre 056, Copenhagen University Hospital Hvidovre, Kettegaard Allé 30, Hvidovre, Copenhagen DK-2650, Denmark; line.jee. hartmann.rasmussen@regionh. dk

Received 5 October 2015 Accepted 26 July 2016 Published Online First

2 September 2016

\begin{abstract}
Objective Soluble urokinase plasminogen activator receptor (suPAR) is an inflammatory biomarker associated with presence and progression of disease and with increased risk of mortality. We aimed to evaluate the unspecific biomarker suPAR as a prognostic marker in patients admitted to acute care.
\end{abstract}

Methods This registry-based retrospective cohort study included 4343 consecutively admitted patients from the Acute Medical Unit at a large Danish university hospital. Time to readmission and death were analysed by multiple Cox regression. Results were reported as HRs for 30-day and 90-day follow-up.

Results During 30-day follow-up, 782 patients $(18.0 \%)$ were readmitted and 224 patients $(5.2 \%)$ died. Comparing 30-day readmission and mortality between patients in the highest and lowest suPAR quartiles yielded $\mathrm{HRs}$ of $2.11(95 \% \mathrm{Cl} 1.70$ to 2.62$)$ and 4.11 (95\% Cl 2.46 to 6.85$)$, respectively, when adjusting for age, sex, Charlson score and $\mathrm{C}$ reactive protein. Area under the curve for receiver operating characteristics curve analysis of suPAR for 30-day mortality was 0.84 (95\% Cl 0.81 to 0.86). Furthermore, in the entire cohort, women had slightly higher suPAR compared with men, and suPAR was associated with age, admission time, admission to intensive care unit and Charlson score.

Conclusions In this large unselected population of acute medical patients, suPAR is strongly associated with disease severity, readmission and mortality after adjusting for all other risk factors, indicating that suPAR adds information to established prognostic indicators. While patients with low suPAR levels have low risk of readmission and mortality, patients with high suPAR levels have a high risk of adverse events.

\section{INTRODUCTION}

In Denmark, the average admission time and number of beds in medical wards have decreased in recent years, and more patients are treated on an outpatient basis. From 2006 to 2013, the average admission time decreased from 4.2 to 3.2 days, while the annual number of outpatients increased by $18 \% .{ }^{1}$ The development in the Organization for Economic Cooperation and Development countries shows the same trend, although less dramatic; the average admission length decreased from 7.9 days

\section{Key messages}

What is already known on this subject?

- Soluble urokinase plasminogen activator receptor (suPAR) is an unspecific inflammatory biomarker.

- Elevated suPAR levels are associated with presence and progression of disease and increased risk of mortality.

- It remains unknown if the prognostic information conveyed by high suPAR is already obvious from other prognostic indicators and whether suPAR can identify patients at low risk of these outcomes.

What might this study add?

- This retrospective cohort study shows that soluble urokinase plasminogen activator receptor predicts risk of readmission and mortality in unselected acute medical patients after adjustment for known risk factors.

in 2006 to 7.4 days in 2012, while the number of outpatient visits grew by $14 \%{ }^{2}$

There are substantial benefits of shorter admissions, including fewer hospital-acquired infections, ${ }^{3}$ reduced immobilisation ${ }^{4}$ and lower cost. However, the resulting high patient turnover requires a high degree of certainty in diagnoses and risk assessment to reduce the risk of insufficient treatment or care. Patients at risk of getting insufficient treatment include the frail and the elderly, patients with multiple comorbidities and patients with poor communication skills, since their diagnoses and risk may be more unclear. The number of elderly, frail, multimorbid patients is increasing rapidly, challenging our standardised, fast-track approach to medical treatment. ${ }^{5}$ At the same time, some patients admitted to the hospital could instead be treated in the primary sector.

One way to improve the resource allocation is to rapidly and correctly assess the patient's prognosis. Soluble urokinase plasminogen activator receptor (suPAR) is an inflammatory biomarker that can be easily measured in plasma or serum. Several studies of both patients and healthy persons have shown 
that suPAR is an unspecific risk marker in a very broad sense, that is, both for future development of disease, presence and severity of current disease and risk of death. ${ }^{6-10}$ However, it is currently unknown if the prognostic information conveyed by high suPAR is already obvious from other prognostic indicators. Also, it is currently unknown if suPAR is effective at identifying low-risk patients. suPAR was recently implemented as a standard biomarker measured on all acutely admitted medical patients at our hospital.

We aimed to evaluate the prognostic strength of suPAR and investigate if suPAR adds information on patient risk to wellestablished prognostic indicators as sex, age, diagnoses and comorbidities. Our secondary objective was to define high-risk and low-risk groups based on suPAR levels as a basis for clinical guidelines.

\section{METHODS}

\section{Study design and setting}

The study was a registry-based retrospective cohort study conducted in the Acute Medical Unit (AMU) at Copenhagen University Hospital Hvidovre, Capital Region, Denmark. The AMU receives medical patients within all specialties, except children, gastroenterological patients and obstetric patients. All patients admitted to the AMU between 18 November 2013 and 31 March 2014 were included in the study.

In the AMU, all patients have a blood sample drawn upon admission, which is analysed for a set of standard biomarkers, including electrolytes, blood counts, liver function, kidney function and markers of infection and inflammation (see online supplementary table S1). suPAR was added to this standard admission blood test panel on 18 November 2013 and is now routinely measured in all patients upon admission to the AMU.

For each patient, we identified the index admission, defined as the first admission at which suPAR was measured. Follow-up was 90 days. There was a total of 2338 readmissions recorded for this patient cohort during follow-up. Of these, 284 were elective readmissions and were excluded from the dataset. Readmissions were thus defined as any acute, non-elective admission during follow-up.

\section{Data}

Data on plasma suPAR levels and the standard blood tests were extracted from the records of the Department of Clinical Biochemistry. All residents in Denmark are registered in the Danish Civil Registration System with a unique personal identification number, ${ }^{11}$ which also identifies the person in other national registries. From the Civil Registration System, we extracted data on sex, date of birth and vital status during follow-up. The Danish National Patient Registry (NPR) contains information on all hospital admissions, ${ }^{12}$ and we extracted data on dates of admission and discharge, as well as all diagnoses registered with the International Classification of Diseases, 10th edition (ICD-10)-system, including primary cause of admission and comorbid conditions. Admission to the intensive care unit (ICU) was registered in a local hospital registry. Thus, data on suPAR levels from the index admission were linked with data on biochemistry, diagnoses, admissions, readmissions and mortality as well as information on admission to the ICU.

\section{Outcomes and covariates}

The primary outcomes investigated were time to readmission or death within 90 days after admission, and the risk of readmission and death at 30 and 90 days after admission. Secondary outcomes were admission time, admission to ICU and suPAR's association with comorbidities. Covariates comprised sex, age, Charlson score and $\mathrm{C}$ reactive protein (CRP).

\section{Measurement of biomarkers}

Blood samples were analysed at the Department of Clinical Biochemistry. Plasma suPAR levels were determined in singlets using the suPARnostic AUTO Flex ELISA kit on an automated Siemens BEP2000 platform according to the manufacturer's instructions (ViroGates A/S, Birkerød, Denmark). The fresh plasma samples were analysed in batches once daily during weekdays (within 0-72 hours after blood sampling). Actual analysis time was approximately 2.5 hours, and the assay had a precision (coefficient of variation) of $5.1 \%$ at $2 \mathrm{ng} / \mathrm{mL}$ and $1.7 \%$ at $7 \mathrm{ng} / \mathrm{mL}$. The study was conducted in a trial period where no clinical guidelines or reference interval for suPAR were available, and because of the batch analysis of suPAR, the results were not available to the physicians in due time for clinical decisions. CRP was measured using a COBAS 6000 analyser (Roche Diagnostics, Mannheim, Germany), and analysis time was approximately $10 \mathrm{~min}$.

\section{Charlson score}

The Charlson score was calculated for each patient based on the patient's comorbid conditions using a SAS macro (developed by Ken Turner and Charles Burchill) as previously described. Briefly, the score is calculated using a weighted scoring system where severe and multiple comorbidities increase the cumulative score. ${ }^{13}$ Input were all primary and secondary diagnoses registered in the NPR upon discharge from the index admission.

The 17 original Charlson groups were collapsed into 10 groups as previously described.?

\section{Statistical analysis}

The following transformations were applied to the data in order to simplify the interpretation and translatability of the results. To visualise the relationship between suPAR and outcomes, agespecific $(<50,50-70$ and $>70$ years) and sex-specific suPAR quartiles were generated for the survival analyses (see online supplementary table S2). This was done to avoid crowding of patients with high mortality in the upper suPAR quartiles, that is, elderly male patients. As the suPAR results were available with only one decimal and thus contained ties, the groups defined by the suPAR quartiles were not equally sized. Adjusted Cox regression analyses were performed separately for each agespecific and sex-specific suPAR quartile to control for confounding. Finally, to make the results generally translatable to other settings, unadjusted mortality rates were calculated for $3 \mathrm{ng} / \mathrm{mL}$ suPAR intervals based on 30-day mortality, and adjusted Cox regression was performed on $\log 2$-transformed data, as this proved to be a good and parsimonious fit to the data.

Continuous data are presented as medians with IQR. The association between suPAR and categorical variables was analysed with the Kruskal-Wallis test. The association between suPAR and CRP was analysed by linear regression analysis.

To investigate the association between suPAR and comorbidities, median suPAR was calculated for the collapsed Charlson comorbidity groups and compared with median suPAR for patients without comorbidities (Charlson score of 0 ).

Cumulative incidence plots were made for readmission for age-specific and sex-specific suPAR quartiles, using death before readmission as a competing end point.

We used Cox regression analysis to estimate the adjusted effect of age-specific and sex-specific suPAR quartiles and continuous log2-transformed suPAR values on readmission and 
mortality, using time to readmission and time to death as outcomes, respectively. Adjustments were made for age, sex, Charlson score and CRP. Results are presented as HRs with 95\% CIs and $\mathrm{p}$ values.

To assess the discriminative ability of suPAR with regards to mortality, we used the area under the curve (AUC) for receiver operating characteristics (ROC) curves. A cut-off value was determined using Youden's index ${ }^{14}$ and AUCs for different ROC curves were compared with the DeLong test. ${ }^{15}$

Survival for age-specific and sex-specific suPAR quartiles are presented in a Kaplan-Meier plot. Log-rank test was used to compare readmission or survival across suPAR quartiles in cumulative incidence and Kaplan-Meier plots.

SAS Enterprise Guide 5.1 (SAS Institute) and R 3.0.3 (The R Foundation for Statistical Computing) were used for statistical

Table 1 Median suPAR level ( $\mathrm{ng} / \mathrm{mL}$ ) stratified according to demographics, disease severity and outcomes

\begin{tabular}{|c|c|c|c|c|}
\hline Variable & n (\%) & Median & IQR & p Value \\
\hline All & $4343(100)$ & 3.2 & $2.3-4.7$ & \\
\hline \multicolumn{5}{|l|}{ Sex } \\
\hline Male & $2075(47.8)$ & 3.1 & $2.2-4.7$ & \\
\hline Female & $2268(52.2)$ & 3.2 & $2.3-4.7$ & 0.006 \\
\hline \multicolumn{5}{|l|}{ Age (years) } \\
\hline$<50$ & $1339(30.8)$ & 2.3 & $1.8-3.0$ & \\
\hline $50-70$ & $1313(30.2)$ & 3.0 & $2.3-4.2$ & \\
\hline$>70$ & 1691 (38.9) & 4.4 & $3.2-6.1$ & $<0.0001$ \\
\hline \multicolumn{5}{|l|}{ Admission time } \\
\hline 0 days & $2218(51.1)$ & 2.6 & $1.9-3.6$ & \\
\hline 1 day & $565(13.0)$ & 3.2 & $2.3-4.5$ & \\
\hline $2-4$ days & $596(13.7)$ & 3.7 & $2.7-5.3$ & \\
\hline $5-7$ days & $371(8.5)$ & 4.4 & $3.3-6.5$ & \\
\hline $8-9$ days & $131(3.0)$ & 5.0 & $3.4-7.3$ & \\
\hline 10 days or more & 462 (10.6) & 5.1 & $3.6-7.5$ & $<0.0001$ \\
\hline \multicolumn{5}{|l|}{ Admitted to ICU } \\
\hline No & $4255(98.0)$ & 3.2 & $2.2-4.6$ & \\
\hline Yes & $88(2.0)$ & 5.6 & $3.0-7.9$ & $<0.0001$ \\
\hline \multicolumn{5}{|l|}{ Charlson score* } \\
\hline 0 & 3177 (73.2) & 2.9 & $2.1-4.2$ & \\
\hline 1 & $827(19.0)$ & 3.7 & $2.7-5.4$ & \\
\hline 2 & $227(5.2)$ & 5.0 & $3.8-7.2$ & \\
\hline 3 & $65(1.5)$ & 6.1 & $4.6-8.6$ & \\
\hline $4+$ & $47(1.1)$ & 7.2 & $4.8-10.9$ & $<0.0001$ \\
\hline \multicolumn{5}{|c|}{ Readmitted within 30 days } \\
\hline No, alive & $3370(77.6)$ & 3.0 & $2.2-4.2$ & \\
\hline No, dead & $191(4.4)$ & 6.9 & $4.8-9.8$ & \\
\hline 1 readmission & $619(14.3)$ & 3.8 & $2.7-5.5$ & \\
\hline $2+$ readmissions & $163(3.8)$ & 4.1 & $2.9-6.0$ & $<0.0001$ \\
\hline \multicolumn{5}{|c|}{ Readmitted within 90 days } \\
\hline No, alive & $2893(66.6)$ & 2.9 & $2.1-4.1$ & \\
\hline No, dead & $237(5.5)$ & 6.8 & $4.8-9.5$ & \\
\hline 1 readmission & $741(17.1)$ & 3.5 & $2.6-5.1$ & \\
\hline $2+$ readmissions & $472(10.9)$ & 4.0 & $2.8-5.7$ & $<0.0001$ \\
\hline \multicolumn{5}{|l|}{ Died within 30 days } \\
\hline No & $4119(94.8)$ & 3.1 & $2.2-4.5$ & \\
\hline Yes & $224(5.2)$ & 6.8 & $4.7-9.9$ & $<0.0001$ \\
\hline \multicolumn{5}{|l|}{ Died within 90 days } \\
\hline No & 3957 (91.1) & 3.0 & $2.2-4.3$ & \\
\hline Yes & $386(8.9)$ & 6.4 & $4.5-9.1$ & $<0.0001$ \\
\hline
\end{tabular}

analysis. R 3.0.3 was used to create the figures. A p value $<0.05$ was considered to be statistically significant.

\section{RESULTS}

\section{Study population}

Patients were included from the AMU, Copenhagen University Hospital Hvidovre, between 18 November 2013 and 31 March 2014. During the inclusion period, 4713 patients were admitted to the AMU. A total of $370(7.9 \%)$ patients were excluded due to missing suPAR analysis results $(4.2 \%)$, suPAR below the dynamic assay range $(0.2 \%)$ or if data on the index admission could not be identified in national $(2.5 \%)$ or local registries $(1.0 \%)$ (see online supplementary figure S1). The final study population comprised 4343 patients (92.1\%) (see online supplementary figure $\mathrm{S} 1$ ). At the index admission, the median age was 63.3 years (min 11.4, $\max 102.7)$.

\section{suPAR is associated with admission time, admission to ICU and comorbidities}

suPAR was slightly higher in women compared with men and increased with age (table 1). suPAR measured at admission was positively associated with factors describing a complicated hospital stay, including longer admission time and admission to the ICU (table 1). Almost 30\% of the patients had a Charlson score above 0 , and suPAR measured at admission was positively associated with the Charlson score (table 1). All comorbidity groups had higher suPAR levels compared with patients without comorbidities (table 2).

\section{High suPAR is associated with readmission}

During the 30 or 90 days follow-up, 782 (18.0\%) and 1213 (27.9\%) patients were readmitted, respectively. suPAR measured at admission was significantly higher in patients who were readmitted compared with patients who were not readmitted and survived during the same period (table 1). suPAR levels also increased with the number of readmissions (table 1).

The cumulative incidence rate of readmission increased with increasing suPAR quartiles $(p<0.0001)$, even though more patients also died without readmission in the higher suPAR quartiles (figure 1).

\section{High suPAR is associated with mortality}

Patients who died during 30 or 90 days follow-up had significantly higher suPAR at admission compared with patients who survived (table 1 ), and the mortality rate increased with suPAR quartiles (figure 2), with the lowest mortality in the first suPAR quartile and the highest mortality in the fourth suPAR quartile $(\mathrm{p}<0.0001)$.

In ROC curve analyses, suPAR had an AUC of 0.84 (95\% CI 0.81 to 0.86 ) for predicting 30 -day mortality, which was significantly higher than age and sex combined (AUC 0.79 , 95\% CI 0.76 to $0.81, \mathrm{p}=0.002$ ). A suPAR cut-off at $4.5 \mathrm{ng} / \mathrm{mL}$ had sensitivity and specificity of $78.1 \%$ and $74.8 \%$, respectively. A combined model with age, sex and suPAR yielded an AUC of 0.86 (95\% CI 0.84 to 0.88 ) for predicting 30-day mortality, and this was significantly different from the AUCs for age and sex $(\mathrm{p}<0.0001)$ or suPAR $(\mathrm{p}=0.003)$. Unadjusted mortality rates for 30 and 90 days follow-up are presented in table 3 stratified in $3 \mathrm{ng} / \mathrm{mL}$ suPAR intervals in patients below or above 70 years of age. In patients younger than 70 years, the 30 -day mortality was 13 -fold higher in patients with suPAR $>9 \mathrm{ng} / \mathrm{mL}$ compared with the entire group, and in patients older than 70 years, 30-day mortality was 3 -fold higher in patients with suPAR $>9 \mathrm{ng} / \mathrm{mL}$ compared with the entire group. 
Table 2 Median suPAR level $(\mathrm{ng} / \mathrm{mL})$ for various comorbidities

\begin{tabular}{|c|c|c|c|c|c|c|c|c|}
\hline \multirow[b]{2}{*}{ Comorbidity groupt } & \multicolumn{3}{|l|}{ All } & \multicolumn{2}{|l|}{ Survived* } & \multicolumn{3}{|l|}{ Died* } \\
\hline & n (\%)‡ & Median (IQR) & p Value§ & n (\%)ף & Median (IQR) & n (\%)ף & Median (IQR) & p Value** \\
\hline Charlson score 0 & 3177 (73.2) & $2.9(2.1-4.2)$ & & 3079 (96.9) & $2.9(2.1-4.1)$ & $98(3.1)$ & $6.7(4.6-9.4)$ & $<0.0001$ \\
\hline Cancer & $109(2.5)$ & $5.2(4.0-8.3)$ & $<0.0001$ & $74(67.9)$ & $5.0(3.9-6.7)$ & $35(32.1)$ & $7.4(4.6-12.1)$ & 0.003 \\
\hline COPD & $471(10.8)$ & $3.7(2.8-5.4)$ & $<0.0001$ & $433(91.9)$ & $3.6(2.7-5.1)$ & $38(8.1)$ & $6.3(3.8-9.5)$ & $<0.0001$ \\
\hline CVD & $342(7.9)$ & $4.3(3.0-5.9)$ & $<0.0001$ & $303(88.6)$ & $4.2(2.8-5.5)$ & $39(11.4)$ & $6.4(3.8-8.7)$ & $<0.0001$ \\
\hline Dementia & $45(1.0)$ & $4.5(3.5-5.5)$ & $<0.0001$ & $35(77.8)$ & $4.5(3.3-5.5)$ & $10(22.2)$ & $4.9(4.1-8.4)$ & 0.08 \\
\hline Diabetes & $285(6.6)$ & $4.3(3.0-6.6)$ & $<0.0001$ & $266(93.3)$ & $4.2(2.9-6.5)$ & $19(6.7)$ & $6.7(5.6-9.0)$ & 0.0003 \\
\hline HIVIAIDS & $2(0.05)$ & $8.2(5.4-10.9)$ & - & $2(100.0)$ & $8.2(5.4-10.9)$ & $0(0)$ & - & - \\
\hline Liver disease & $40(0.9)$ & $7.7(5.6-11.5)$ & $<0.0001$ & $34(85.0)$ & $7.3(5.2-11.1)$ & $6(15.0)$ & $8.9(7.4-18.7)$ & 0.16 \\
\hline Paraplegia and hemiplegia & $8(0.2)$ & $4.9(3.4-6.6)$ & 0.01 & $7(87.5)$ & $4.8(2.9-6.2)$ & $1(12.5)$ & $7.0(7.0-7.0)$ & 0.12 \\
\hline Peptic ulcer disease & $19(0.4)$ & $4.7(3.6-8.2)$ & $<0.0001$ & $15(79.0)$ & $4.6(3.6-6.5)$ & $4(21.1)$ & $10.7(5.4-17.4)$ & 0.18 \\
\hline Renal disease & $42(1.0)$ & $9.3(6.4-12.3)$ & $<0.0001$ & $36(85.7)$ & $8.0(6.0-11.0)$ & $6(14.3)$ & 17.3 (13.7-19.7) & 0.002 \\
\hline Rheumatic disease & $12(0.3)$ & $6.4(5.7-9.4)$ & $<0.0001$ & $12(100.0)$ & $6.4(5.7-9.4)$ & $0(0)$ & - & - \\
\hline
\end{tabular}

Across all comorbidity groups, median suPAR was higher in patients who died compared with patients who survived (table 2).

\section{suPAR is independently associated with readmission and death}

In univariate analysis, the HR for readmission and mortality increased with higher suPAR quartiles (table 4). In the multiple analysis, adjusted for age, sex and Charlson score, the HR for readmission and mortality remained higher in the highest suPAR quartile compared with the lowest, also when CRP was included in the adjusted analysis (table 4).

When adjusted for age, sex and Charlson score, the logtransformed suPAR values gave a HR for 30-day mortality for doubling the suPAR values of 3.07 (95\% CI 2.60 to 3.64,

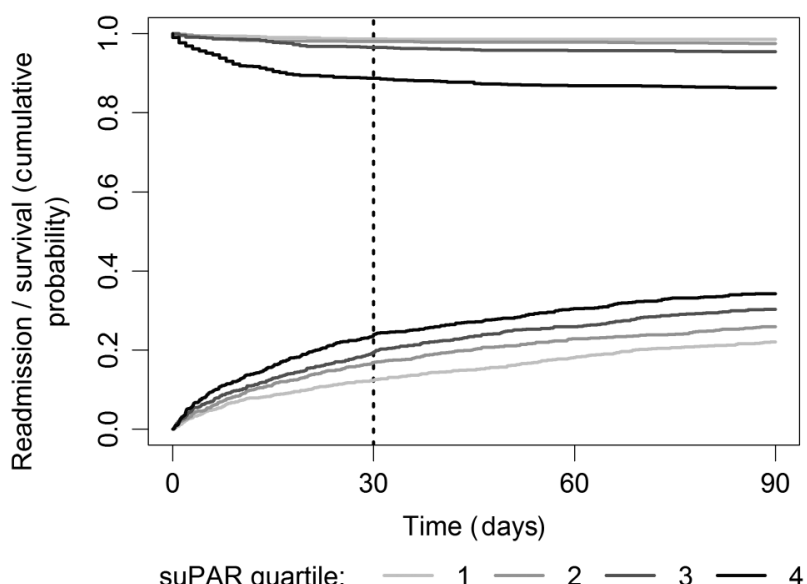

Figure 1 Cumulative incidence of readmission with death without readmission as a competing risk in age-specific and sex-specific soluble urokinase plasminogen activator receptor (suPAR) quartiles within 90 days. The top curves illustrate survival in non-readmitted patients (log-rank test $p<0.0001$ ), and the bottom curves illustrate cumulative incidence of readmission (log-rank test $p<0.0001$ ). $\mathrm{p}<0.0001$ ). When CRP was included in the adjusted analysis, the HR for 30-day mortality for doubling suPAR values was 2.53 (95\% CI 2.10 to $3.04, \mathrm{p}<0.0001)$. All estimates in the full model are summarised in online supplementary table S3.

\section{Relationship between suPAR and CRP}

Even though there was a positive correlation between the inflammatory markers suPAR and CRP $(n=4267$, Kendall's tau-b $0.36, \mathrm{p}<0.0001), 8.4 \%$ of the patients had high suPAR $(>4.5 \mathrm{ng} / \mathrm{mL})$ but low CRP levels $(<10 \mathrm{mg} / \mathrm{l})$. In patients with low CRP $(n=2500)$, suPAR remained predictive of 30-day readmission (HR 1.42 per $\log 2$ increase in suPAR, 95\% CI 1.20 to $1.67, \mathrm{p}<0.0001$ ) and 90 -day mortality (HR 1.63 per $\log 2$ increase in suPAR, 95\% CI 1.15 to $2.30, \mathrm{p} 0.006$ ) after adjustment for age, sex, Charlson score and CRP.

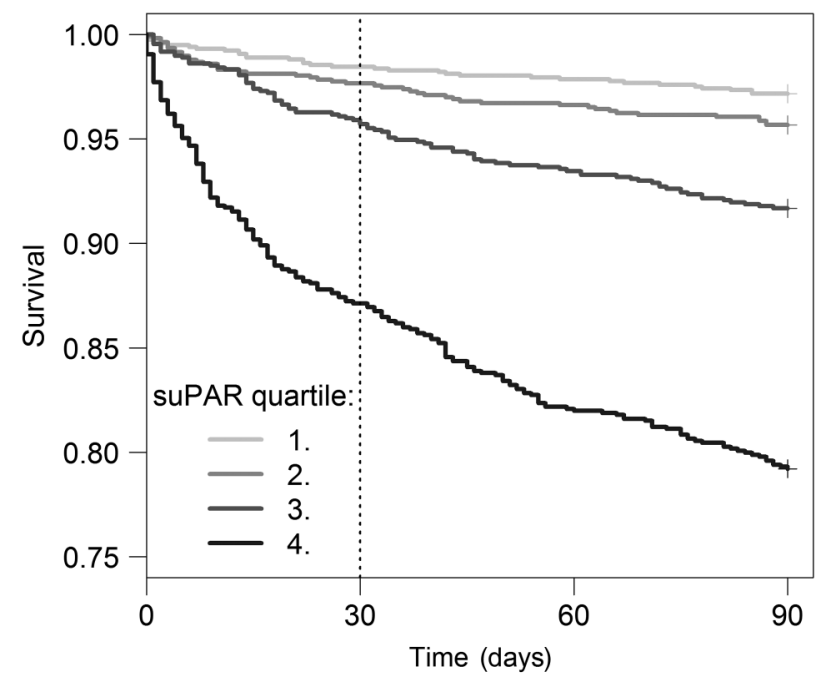

Figure 2 Kaplan-Meier plot of 90-day survival for age-specific and sex-specific soluble urokinase plasminogen activator receptor (suPAR) quartiles. Log-rank test $p<0.0001$. 
Table 3 Mortality risk according to $3 \mathrm{ng} / \mathrm{mL}$ suPAR intervals in patients aged $\leq 70$ years or $>70$ years

\begin{tabular}{|c|c|c|c|c|c|}
\hline \multirow[b]{2}{*}{$\begin{array}{l}\text { suPAR } \\
(\mathrm{ng} / \mathrm{mL})\end{array}$} & \multirow[b]{2}{*}{$\begin{array}{l}\% \text { in } \\
\text { interval (n) }\end{array}$} & \multicolumn{2}{|l|}{30 days } & \multicolumn{2}{|l|}{90 days } \\
\hline & & $\begin{array}{l}\text { Mortality } \\
\%(n)\end{array}$ & $95 \% \mathrm{Cl}^{*}$ & $\begin{array}{l}\text { Mortality } \\
\%(n)\end{array}$ & $95 \% \mathrm{Cl}^{*}$ \\
\hline \multicolumn{6}{|c|}{$\leq 70$ years } \\
\hline All & $100.0(2652)$ & $1.5(41)$ & 1.1 to 2.0 & $2.9(77)$ & 2.2 to 3.6 \\
\hline $0-3$ & 64.3 (1705) & $0.3(5)$ & 0.1 to 0.7 & $0.8(14)$ & 0.5 to 1.4 \\
\hline $3-6$ & $28.1(744)$ & $1.6(12)$ & 0.8 to 2.8 & $3.6(27)$ & 2.4 to 5.2 \\
\hline $6-9$ & $4.8(127)$ & $7.1(9)$ & 3.3 to 13.0 & $11.8(15)$ & 6.8 to 18.7 \\
\hline$>9$ & $2.9(76)$ & $19.7(15)$ & 11.5 to 30.5 & $27.6(21)$ & 18.0 to 39.1 \\
\hline \multicolumn{6}{|c|}{$>70$ years } \\
\hline All & $100.0(1691)$ & $10.8(183)$ & 9.3 to 12.3 & $18.3(309)$ & 16.4 to 20.1 \\
\hline $0-3$ & $20.0(339)$ & $2.7(9)$ & 1.2 to 5.0 & $3.5(12)$ & 1.8 to 6.1 \\
\hline $3-6$ & $54.0(913)$ & $7.1(65)$ & 5.5 to 9.0 & 13.7 (125) & 11.5 to 16.1 \\
\hline $6-9$ & $17.3(293)$ & $19.8(58)$ & 15.4 to 24.8 & $32.8(96)$ & 27.4 to 38.5 \\
\hline$>9$ & $8.6(146)$ & $34.9(51)$ & 27.2 to 43.3 & $52.1(76)$ & 43.6 to 60.4 \\
\hline
\end{tabular}

${ }^{*} \mathrm{Cls}$ were calculated using the Wald method.

suPAR, soluble urokinase plasminogen activator receptor.

\section{DISCUSSION}

In this retrospective cohort study, we investigated the newly introduced inflammatory biomarker suPAR in 4343 consecutively admitted, unselected acute medical patients from the AMU at Copenhagen University Hospital Hvidovre. We found that suPAR was strongly associated with readmissions and allcause mortality. We observed an association between suPAR levels and admission to the ICU. Furthermore, our findings confirmed that suPAR increased with age, admission time and number and severity of comorbidities (Charlson score).
To our knowledge, this study is the largest study of suPAR in an unselected cohort of acute medical patients. Our results support previous findings from acute care; results from a smaller cohort at the same AMU showed the same associations between suPAR and mortality, admission time and Charlson score. Another study, carried out in an Austrian acute care setting, investigated patients suspected for blood infections and found a similar strong prognostic value of suPAR with regard to all-cause mortality. ${ }^{16}$ The present study differs from previous studies by including all acute medical patients regardless of diagnosis and time of day or weekday, thus preventing selection bias.

As follow-up data were extracted from national registries, there was no loss to follow-up. We clearly show that elevated suPAR levels are associated with high risk of short-term mortality, and furthermore, we found a strong association between suPAR and non-elective readmissions, both within 30 and 90 days, and suPAR increased with the number of readmissions. Two Danish studies have shown conflicting results with regard to suPAR and readmission. In our previous study from the AMU, there was no association between suPAR and readmissions, possibly as a result of the smaller sample size or the inclusion process, where there was a potential selection bias towards exclusion of very old or ill patients. ${ }^{7}$ Another recent study including 1036 acutely admitted patients reported a significant relation between suPAR and readmission with an approximately twofold HR in the highest suPAR tertile compared with the lowest suPAR tertile. ${ }^{17}$ Non-elective readmissions constitute an increasing problem of multifactorial origin. Readmissions may occur if patients are discharged from the hospital prematurely or discharged to inappropriate settings, or do not receive adequate information or resources to ensure continued improvement. In addition, lack of coordinated care and communication between hospital-based and community-based providers may

Table 4 HRs in age-specific and sex-specific suPAR quartiles

\begin{tabular}{|c|c|c|c|c|c|c|}
\hline \multirow[b]{2}{*}{ Outcome } & \multicolumn{2}{|l|}{ Univariate } & \multicolumn{2}{|c|}{ Adjusted for age, sex and Charlson } & \multicolumn{2}{|c|}{$\begin{array}{l}\text { Adjusted for age, sex, Charlson and } \\
\text { CRP }\end{array}$} \\
\hline & $\mathrm{HR}(95 \% \mathrm{Cl})$ & p Value & $\mathrm{HR}(95 \% \mathrm{Cl})$ & p Value & $\mathrm{HR}(95 \% \mathrm{Cl})$ & p Value \\
\hline \multicolumn{7}{|c|}{ Readmitted within 30 days } \\
\hline 1. quartile & 1 & $<0.0001^{*}$ & 1 & $<0.0001^{*}$ & 1 & $<0.0001^{*}$ \\
\hline 2. quartile & 1.40 (1.12 to 1.74$)$ & 0.003 & 1.36 (1.09 to 1.69$)$ & 0.007 & 1.39 (1.11 to 1.73$)$ & 0.004 \\
\hline 3. quartile & 1.64 (1.33 to 2.03$)$ & $<0.0001$ & 1.52 (1.22 to 1.88$)$ & 0.0001 & 1.56 (1.25 to 1.94$)$ & $<0.0001$ \\
\hline 4. quartile & 2.22 (1.81 to 2.73$)$ & $<0.0001$ & 2.02 (1.64 to 2.49$)$ & $<0.0001$ & 2.11 (1.70 to 2.62$)$ & $<0.0001$ \\
\hline \multicolumn{7}{|c|}{ Readmitted within 90 days } \\
\hline 1. quartile & 1 & $<0.0001^{*}$ & 1 & $<0.0001^{*}$ & & $<0.0001^{*}$ \\
\hline 2. quartile & 1.23 (1.04 to 1.46$)$ & 0.02 & 1.20 (1.01 to 1.42$)$ & 0.04 & 1.22 (1.03 to 1.45$)$ & 0.02 \\
\hline 3. quartile & 1.49 (1.27 to 1.76$)$ & $<0.0001$ & 1.39 (1.18 to 1.65$)$ & $<0.0001$ & $1.43(1.21$ to 1.70$)$ & $<0.0001$ \\
\hline 4. quartile & 1.92 (1.63 to 2.25$)$ & $<0.0001$ & $1.78(1.51$ to 2.10$)$ & $<0.0001$ & $1.87(1.58$ to 2.21$)$ & $<0.0001$ \\
\hline \multicolumn{7}{|c|}{ Died within 30 days } \\
\hline 1. quartile & 1 & $<0.0001^{*}$ & 1 & $<0.0001^{*}$ & 1 & $<0.0001^{*}$ \\
\hline 2. quartile & $1.52(0.83$ to 2.79$)$ & 0.17 & $1.33(0.73$ to 2.44$)$ & 0.35 & $1.20(0.65$ to 2.21$)$ & 0.57 \\
\hline 3. quartile & 2.80 (1.62 to 4.83$)$ & 0.0002 & 1.92 (1.11 to 3.32$)$ & 0.02 & $1.60(0.92$ to 2.79$)$ & 0.10 \\
\hline 4. quartile & 8.85 (5.41 to 14.47$)$ & $<0.0001$ & 6.06 (3.69 to 9.95$)$ & $<0.0001$ & 4.11 (2.46 to 6.85$)$ & $<0.0001$ \\
\hline \multicolumn{7}{|c|}{ Died within 90 days } \\
\hline 1. quartile & 1 & $<0.0001$ * & 1 & $<0.0001^{*}$ & 1 & $<0.0001^{*}$ \\
\hline 2. quartile & $1.54(0.98$ to 2.40$)$ & 0.06 & 1.35 (0.86 to 2.11$)$ & 0.19 & 1.27 (0.81 to 1.99$)$ & 0.30 \\
\hline 3. quartile & 3.00 (2.01 to 4.47$)$ & $<0.0001$ & $2.10(1.41$ to 3.14$)$ & 0.0003 & 1.88 (1.25 to 2.82$)$ & 0.002 \\
\hline 4. quartile & $8.13(5.64$ to 11.72$)$ & $<0.0001$ & 5.83 (4.03 to 8.43 ) & $<0.0001$ & 4.54 (3.11 to 6.63 ) & $<0.0001$ \\
\hline
\end{tabular}


also lead to unplanned readmissions. A marker of readmission might aid in the identification of patients in need of additional clinical attention to finalise treatment before discharge or to initiate a cross-sectorial intervention, in order to prevent future readmissions. However, to properly assess whether suPAR could be a useful measure of readmission in the clinic, the suPAR measurement should have been carried out at discharge rather than at admission, as the patient status may have changed during the admission. Thus, the association we found between high suPAR and readmission at the time of admission may not be clinically applicable per se, but support that suPAR is a surrogate marker of disease severity or additional underlying disease and could raise awareness of morbidity other than the acute illness already from the point of admission.

The challenge for the clinical implementation of suPAR is the unspecific nature of this prognostic marker, which makes it difficult to define one single intervention or action to apply to patients with a high suPAR in an unselected patient population. However, considering the high risk connected with increased suPAR levels, clinical reconsideration is advised when encountering a patient with an unexplained high suPAR, in which case an individual intervention should be scheduled based on symptoms and objective findings for the particular clinical issue, for example, referral to a specialist, follow-up consultation at general practitioner, positron emission tomography scan or other diagnostic procedures. On the other hand, a low suPAR might promote faster discharge. In this way, the mere information of patient prognosis, provided by suPAR, may influence clinical decisions and thereby possibly have impact on outcome. However, it should be emphasised that currently there are no data to support that knowledge of the prognosis of a particular patient can actually lead to a favourable change in outcome. Some studies show how various treatments lowers the suPAR level along with a clinical improvement in the particular condition, for example, cervical cancer, leukaemia or malaria, but do not investigate whether this is also associated with an improved prognosis. ${ }^{18-20}$ Whether or not knowledge of suPAR at time of admission to the ED can impact on outcome is currently being investigated in a randomised intervention study at two large hospitals in the capital region of Denmark (ClinicalTrials.gov number, NCT02643459).

The Charlson score is an example of a strong prognostic tool, which accurately predicts a patient's risk of mortality. ${ }^{21}$ Median suPAR levels were increased across all comorbidity groups compared with patients who had not been diagnosed with any predefined chronic comorbid condition, and suPAR increased with increasing Charlson score, indicating that suPAR is indeed a marker of disease severity. In support of this, increased suPAR predicts new-onset cardiovascular complications in patients with other chronic diseases. ${ }^{22} 23$ Even within individual comorbidity groups, survivors had lower suPAR than non-survivors. This ability of suPAR to further stratify the Charlson score with regards to mortality underlines the strength of suPAR as a prognostic tool.

Similarly, when we adjusted for another prognostic biomarker, CRP, suPAR remained independently associated with outcome, even in the group of patients with low CRP. Although suPAR and CRP are both inflammatory biomarkers, they have been suggested to reflect different aspects of inflammation with suPAR representing inflammation at the cellular level and CRP representing inflammation at the metabolic level. ${ }^{24} 25$ The combined information of suPAR and CRP significantly increases the Framingham Risk Score's ability to predict cardiovascular disease and mortality in the general population. ${ }^{26}$
The suPAR levels were particularly high in patients with liver or kidney disease. Although the number of patients in these two groups was low, the results are supported by other studies of patients with chronic liver or kidney disease. ${ }^{23} 27$ Aside from indicating a high risk of readmission or death independent of the diagnosis, suPAR also reflects the total comorbidity burden. Thus, if a patient assumed to have a low disease burden presents with high suPAR levels, it should bring attention to possibly unidentified diseases, such as liver or kidney disease, in addition to immediate acute diagnoses.

The trend towards fewer hospital beds, shorter admissions and more elderly and chronically ill patients in addition to financial constraints on the healthcare sector, results in more fast-track patient courses and shorter time for clinical investigation and diagnostics. An objective marker of disease presence and severity may aid the clinician in evaluating whether a patient is severely ill or not. This may also be of interest for the general practitioner or in the prehospital setting.

An efficient admission process is crucial to quickly identify high-risk patients who need urgent clinical attention and also to identify low-risk patients who may be discharged and thereby avoid unnecessary and potentially harmful in-hospital stays. Furthermore, a Danish study recently demonstrated how high bed occupancy rates were associated with increased inpatient and 30-day mortality. ${ }^{28}$ Improved risk assessment may help identify patients suitable for outpatient treatment and thus decrease bed occupancy. Our study shows that a low suPAR can identify patients with an even lower mortality than the average, who may be suitable for treatment outside the hospitals. While using the adjusted Cox regression analyses may be the most accurate way to use suPAR for prognostication, the unadjusted mortality risks with $95 \% \mathrm{CI}$ in table 3 presents a simple way in which a patient's risk can be estimated and added to the overall clinical assessment.

Our results indicate that suPAR adds significant prognostic information to other well-established prognostic measures and indicators, including age, sex, Charlson score and CRP, but whether it is superior to the prognostic assessment made by the medical staff remains unknown. We assume that suPAR could be a valuable addition to the existing prognostic tools and indicators as this biomarker imposes a very simple way to give a prognostic risk estimate. Thus, it could perform as an 'attention biomarker', which mostly provides supporting information to the clinical assessments made by the staff, but sometimes presents unexpected high or low values for a given patient, prompting changes in the clinical investigation or treatment.

\section{Limitations}

A limitation to the study is the lack of data on smoking, as suPAR is confounded by smoking. ${ }^{29}$ Furthermore, the NPR is based on the registration of ICD-10 diagnoses at the end of an admission. The credibility of the registry has been validated, and the positive predictive value of the coding of Charlson comorbidities was found to be very high; ${ }^{30}$ however, registration may be inadequate and underlying diagnoses might not be completely registered on every admission. Diagnoses that results in economic reimbursement for the admitting departments may also become more consistently registered. The suPAR results were not available to the doctors in the AMU as the suPAR test was run in batches only once daily. However, suPAR may have been available in the electronic blood sample database to the doctors at the specialised departments, and we cannot exclude that it can have influenced clinical decisions here. As previously mentioned, another limitation to the study is the lack of suPAR 
measurements at time of discharge. This would be necessary to properly assess if suPAR could be used in the clinic as a marker of readmission.

\section{CONCLUSION}

In conclusion, a high suPAR level at admission to the AMU is a marker of severe disease and associated with increased risk of readmission and mortality. Plasma suPAR levels may therefore provide benefit for evaluation of medical patients admitted to the AMU to determine the requirement for a more extensive clinical assessment and more intensive monitoring and care.

Contributors LJHR, SL, THH, JE-O and OA conceived and coordinated the investigation. LJHR and SL performed the statistical analyses. LJHR, SL, THH, JE-O and $\mathrm{OA}$ interpreted the data. LJHR, SL, THH, JE-O and OA drafted the manuscript. LJHR, SL, THH, GE, JHP, KI, JE-O and OA contributed to the discussion and reviewed and edited the article. LJHR is the guarantor of the study.

Funding This study received no external funding, but LJHR is supported by a grant from the Lundbeck Foundation (grant no. R180-2014-3360).

Competing interests LJHR has received funding for travel from ViroGates $A / S$, Denmark, the company that produces the suPARnostic assays. JE-O and OA are named inventors on patents on suPAR and prognosis. The patents are owned by Copenhagen University Hospital Hvidovre, Denmark, and licensed to ViroGates A/S. $\mathrm{JE}-\mathrm{O}$ is a co-founder, shareholder and CSO of ViroGates A/S.

Ethics approval This registry-based study was approved by the Danish Health and Medicines authority (reference no. 3-3013-1061/1). All processing of personal data followed national guidelines, and the project was approved by the Danish Data Protection Agency (reference no. HVH-2014-018, 02767).

Provenance and peer review Not commissioned; externally peer reviewed.

Open Access This is an Open Access article distributed in accordance with the Creative Commons Attribution Non Commercial (CC BY-NC 4.0) license, which permits others to distribute, remix, adapt, build upon this work non-commercially, and license their derivative works on different terms, provided the original work is properly cited and the use is non-commercial. See: http://creativecommons.org/ licenses/by-nc/4.0/

\section{REFERENCES}

Statistics Denmark. http://www.dst.dk/en

2 OECD.Stat. http://stats.oecd.org/

3 Rosman M, Rachminov 0, Segal O, et al. Prolonged patients' In-Hospital Waiting Period after discharge eligibility is associated with increased risk of infection, morbidity and mortality: a retrospective cohort analysis. BMC Health Serv Res 2015;15:246.

4 Pedersen MM, Bodilsen AC, Petersen J, et al. Twenty-four-hour mobility during acute hospitalization in older medical patients. J Gerontol A Biol Sci Med Sci 2013;68:331-7.

5 Boyd CM, Darer J, Boult C, et al. Clinical practice guidelines and quality of care for older patients with multiple comorbid diseases: implications for pay for performance. JAMA 2005;294:716-24.

6 Eugen-Olsen J, Andersen 0, Linneberg A, et al. Circulating soluble urokinase plasminogen activator receptor predicts cancer, cardiovascular disease, diabetes and mortality in the general population. J Intern Med 2010;268:296-308.

7 Haupt TH, Petersen J, Ellekilde G, et al. Plasma suPAR levels are associated with mortality, admission time, and Charlson Comorbidity Index in the acutely admitted medical patient: a prospective observational study. Crit Care 2012;16:R130.
8 Thunø M, Macho B, Eugen-Olsen J. suPAR: the molecular crystal ball. Dis Markers 2009:27:157-72.

9 Koch A, Tacke F. Risk stratification and triage in the emergency department: has this become 'suPAR' easy? J Intern Med 2012;272:243-6.

10 Hayek SS, Sever $S$, Ko YA, et al. Soluble urokinase receptor and chronic kidney disease. N Engl J Med 2015:373:1916-25.

11 Pedersen CB. The Danish Civil Registration System. Scand J Public Health 2011:39:22-5.

12 Lynge E, Sandegaard JL, Rebolj M. The Danish National Patient Register. Scand J Public Health 2011:39:30-3.

13 Charlson ME, Pompei $\mathrm{P}$, Ales $\mathrm{KL}$, et al. A new method of classifying prognostic comorbidity in longitudinal studies: development and validation. J Chronic Dis 1987:40:373-83.

14 Youden WJ. Index for rating diagnostic tests. Cancer 1950;3:32-5.

15 DeLong ER, DeLong DM, Clarke-Pearson DL. Comparing the areas under two or more correlated receiver operating characteristic curves: a nonparametric approach. Biometrics 1988;44:837-45.

16 Raggam RB, Wagner J, Prüller $F$, et al. Soluble urokinase plasminogen activator receptor predicts mortality in patients with systemic inflammatory response syndrome. J Intern Med 2014;276:651-8.

17 Nayak RK, Allingstrup M, Phanareth K, et al. suPAR as a biomarker for risk of readmission and mortality in the acute medical setting. Dan Med J 2015;62:A5146

18 Perch M, Kofoed PE, Fischer TK, et al. Serum levels of soluble urokinase plasminogen activator receptor is associated with parasitemia in children with acute Plasmodium falciparum malaria infection. Parasite Immunol 2004:26:207-11.

19 Mustjoki S, Sidenius N, Sier CFM, et al. Soluble urokinase receptor levels correlate with number of circulating tumor cells in acute myeloid leukemia and decrease rapidly during chemotherapy. Cancer Res 2000;60:7126-32.

20 Jing J, Zheng S, Han C, et al. Evaluating the value of uPAR of serum and tissue on patients with cervical cancer. J Clin Lab Anal 2012;26:16-21.

21 Frenkel WJ, Jongerius EJ, Mandjes-van Uitert MJ, et al. Validation of the Charlson Comorbidity Index in acutely hospitalized elderly adults: a prospective cohort study. J Am Geriatr Soc 2014;62:342-6.

22 Rasmussen L, Knudsen A, Katzenstein $T L$, et al. Soluble urokinase plasminogen activator receptor (suPAR) is a novel, independent predictive marker of myocardial infarction in HIV-1-infected patients: a nested case-control study. HIV Med 2016:17:350-7

23 Meijers B, Poesen $\mathrm{R}$, Claes $\mathrm{K}$, et al. Soluble urokinase receptor is a biomarker of cardiovascular disease in chronic kidney disease. Kidney Int 2015;87:210-16.

24 Lyngbæk S, Sehestedt T, Marott JL, et al. CRP and suPAR are differently related to anthropometry and subclinical organ damage. Int J Cardiol 2013;167:781-5.

25 Hodges GW, Bang CN, Wachtell K, et al. suPAR: a new biomarker for cardiovascular disease? Can J Cardiol 2015:31:1293-302.

26 Lyngbæk S, Marott JL, Sehestedt T, et al. Cardiovascular risk prediction in the general population with use of suPAR, CRP, and Framingham Risk Score. Int J Cardiol 2013;167:2904-11.

27 Zimmermann HW, Koch A, Seidler S, et al. Circulating soluble urokinase plasminogen activator is elevated in patients with chronic liver disease, discriminates stage and aetiology of cirrhosis and predicts prognosis. Liver Int 2012:32:500-9.

28 Madsen F, Ladelund S, Linneberg A. High levels of bed occupancy associated with increased inpatient and thirty-day hospital mortality in Denmark. Health Aff (Millwood) 2014;33:1236-44.

29 Haupt TH, Kallemose T, Ladelund S, et al. Risk factors associated with serum levels of the inflammatory biomarker soluble urokinase plasminogen activator receptor in a general population. Biomark Insights 2014;9:91-100.

30 Thygesen SK, Christiansen CF, Christensen S, et al. The predictive value of ICD-10 diagnostic coding used to assess Charlson comorbidity index conditions in the population-based Danish National Registry of Patients. BMC Med Res Methodol 2011;11:83. 